\title{
VALUE DISTRIBUTION AND POWER SERIES WITH MODERATE GAPS
}

\author{
L. R. Sons
}

\section{INTRODUCTION}

For entire functions it is known (see [1], [3], and [5]) that certain assumptions on the gaps in the power series expansion of the function about zero imply that the function has not only one zero (or a-value) but infinitely many. To obtain corresponding results for functions analytic in the unit disk, it is necessary to link the gap assumption with a growth assumption on the function.

THEOREM 1 (Nevanlinna's notation [4, pp. 4 and 18]). Let

$$
f(z)=\sum_{k=0}^{\infty} c_{k} z^{n_{k}}
$$

be analytic in $|\mathrm{z}|<1$, with $\mathrm{n}_{0}=0$. Let $\mathrm{N}^{0}(\mathrm{t})$ be the number of $\mathrm{n}_{\mathrm{k}}$ not greater than t. If for some fixed $\beta(0<\beta<1)$

$$
N^{0}(t)=O\left(t^{1-\beta}\right) \quad(t \rightarrow \infty),
$$

and if

$$
n(r, 1 / f)=O\left((1-r)^{-\lambda}\right) \quad(r \rightarrow 1)
$$

then

$$
\log \mathrm{M}(\mathrm{r})=\mathrm{O}\left((1-\mathrm{r})^{-\alpha}\right) \quad(\mathrm{r} \rightarrow 1)
$$

for every $\alpha$ with

$$
\alpha>\max \left(\lambda, \frac{1-\beta}{\beta}\right)
$$

COROLLARY. Let $\mathrm{f}(\mathrm{z})$ be analytic in $|\mathrm{z}|<1$ and of the form (1), with $\mathrm{n}_{0}=0$ and the $\mathrm{n}_{\mathrm{k}}$ satisfying (2). If

$$
\underset{r \rightarrow 1}{\lim \sup } \frac{\log \log M(r)}{-\log (1-r)} \geq \alpha \quad\left(\alpha>\frac{1-\beta}{\beta}\right)
$$

then

$$
\limsup _{r \rightarrow 1} \frac{\log n(r, 1 / f)}{-\log (1-r)} \geq \alpha
$$

Theorem 1, which has an elementary proof, extends a theorem stated by $F$. Sunyer I. Balaguer [7]. Related problems in the disk have recently been investigated for larger gaps-Hadamard gaps-by G. and M. Weiss [8] and Ch. Pommerenke [6]. 
A function satisfying the gap condition of Theorem 1 is the theta-function

$$
1+\sum_{n=1}^{\infty} 2 z^{n^{2}}=\prod_{n=1}^{\infty}\left(1+z^{2 n-1}\right)^{2} \cdot\left(1-z^{2 n}\right) .
$$

It has $M(r)=O\left((1-r)^{-1 / 2}\right)$.

\section{STATEMENTS OF PRELIMINARY LEMMAS}

The following two lemmas form the basis of the proof of Theorem 1; we shall prove them in Section 4.

LEMMA 1. If $0<\varepsilon<1$ and $\phi(\mathrm{x})$ is a continuous, increasing function in $0<\mathrm{x}<1$ such that

$$
\phi\left(\mathrm{x}_{0}\right)>\frac{4}{\varepsilon\left(1-\mathrm{x}_{0}\right)}
$$

for some $\mathrm{x}_{0}\left(0<\mathrm{x}_{0}<1\right)$, then there exists an $\mathrm{x}^{\prime}\left(\mathrm{x}_{0} \leq \mathrm{x}^{\prime}<\left(1+\mathrm{x}_{0}\right) / 2\right)$ such that

$$
\phi\left(\mathrm{x}^{\prime}+\frac{1}{\phi\left(\mathrm{x}^{\prime}\right)}\right)<(1+\varepsilon) \phi\left(\mathrm{x}^{\prime}\right) \text {. }
$$

LEMMA 2. Let $\left\{\mathrm{n}_{\mathrm{k}}\right\}$ be a strictly increasing sequence of integers satisfying (2). For each sequence $\varepsilon_{0}, \varepsilon_{1}, \varepsilon_{2}, \cdots$ with $\varepsilon_{\mathrm{k}}= \pm 1$, there exists a real-valued function $\mathrm{g}(\mathrm{t})$ in $0 \leq \mathrm{t} \leq 1$ such that

(i) $\varepsilon_{k} \int_{0}^{l} g(t) t^{n_{k}} d t=A_{k}>0$,

(ii) $\int_{0}^{1}|g(t)| d t \leq 1 / 2$,

(iii) $\inf _{\mathrm{k}} \mathrm{A}_{\mathrm{k}} \rho^{-\mathrm{n}_{\mathrm{k}}}>\exp \left(-\mathrm{K}(1-\rho)^{-(1-\beta) / \beta} \log ^{1 / \beta}(1 /(1-\rho))\right)$

for $\mathrm{R}_{0}<\rho<1$, where $\mathrm{K}$ and $\mathrm{R}_{0}$ are independent of the sequence $\left\{\varepsilon_{\mathrm{k}}\right\}$.

Lemma 1 goes back essentially to Borel.

\section{PROOF OF THEOREM 1}

First we estimate

$$
U(r)=\sup _{0 \leq \mathrm{u} \leq \mathrm{r}}|\mathrm{f}(\mathrm{u})|
$$

from below. Let

$$
\varepsilon_{\mathrm{k}}=\operatorname{sign}\left(\Re \mathrm{c}_{\mathrm{k}}\right) \text {, }
$$


where

$$
\operatorname{sign} v=\left\{\begin{array}{cl}
1 & \text { if } v \geq 0 \\
-1 & \text { if } v<0
\end{array}\right.
$$

Construct the function $\mathrm{g}(\mathrm{t})$ of Lemma 2 for this choice of $\left\{\varepsilon_{\mathrm{k}}\right\}$. Then, for $\mathrm{R}_{0}<\rho<1$

$$
\begin{aligned}
\exp \left(-\mathrm{K}(1-\rho)^{-(1-\beta) / \beta}\right. & \left.\log ^{1 / \beta}(1 /(1-\rho))\right) \cdot \sum\left|\Re c_{k}(\rho r)^{n k}\right| \\
& \leq\left\{\inf _{\mathrm{k}} A_{k} \rho^{-n_{k}}\right\} \cdot \sum\left|\Re c_{k}(\rho r)^{n_{k}}\right| \\
& \leq \sum\left|\Re c_{k}(\rho r)^{n_{k}}\right| A_{k} \rho^{-n_{k}} .
\end{aligned}
$$

The right-hand side is equal to

$$
\begin{aligned}
\sum \Re c_{k} r^{n_{k}} \int_{0}^{l} t^{n_{k}} g(t) d t & =\Re \int_{0}^{l} f(r t) g(t) d t \\
& \leq \int_{0}^{l}|f(r t)||g(t)| d t \leq U(r) \int_{0}^{l}|g(t)| d t \leq \frac{1}{2} U(r)
\end{aligned}
$$

By the same argument, for $R_{0}<\rho<1$,

$$
\exp \left(-\mathrm{K}(1-\rho)^{-(1-\beta) / \beta} \log ^{\mathrm{l} / \beta}(1 /(1-\rho))\right) \cdot \sum\left|\mathfrak{s}_{\mathrm{k}}(\rho \mathrm{r})^{\mathrm{n}_{\mathrm{k}}}\right| \leq \frac{1}{2} \mathrm{U}(\mathrm{r})
$$

But

$$
\sum\left|\Re c_{k}(\rho r)^{n_{k}}\right|+\sum\left|\Im c_{k}(\rho r)^{n k}\right| \geq \sum\left|c_{k}(\rho r)^{n k}\right| \geq M(\rho r)
$$

Therefore, adding (6) and the corresponding inequality for the real parts of the $c_{k}$, we obtain the inequality

$$
\mathrm{U}(\mathrm{r}) \geq \exp \left(-\mathrm{K}(1-\rho)^{-(1-\beta) / \beta} \log ^{1 / \beta}(1 /(1-\rho))\right) \cdot \mathrm{M}(\rho \mathrm{r}) .
$$

By considering $f\left(u e^{i \theta}\right)$ in place of $f(u)$ and noting in (iii) of Lemma 2 that $K$ and $R_{0}$ are independent of the sequence $\left\{\varepsilon_{k}\right\}$, we see that if $\varepsilon^{\prime}>0$, then (7) implies that there exists a value $\rho_{0}$, independent of $\theta$, such that the inequalities

$$
\begin{aligned}
\sup _{\mathrm{u} \leq \mathrm{r}}\left|\mathrm{f}\left(\mathrm{ue}^{\mathrm{i} \theta}\right)\right| & \geq \exp \left(-\mathrm{K}(1-\rho)^{-(1-\beta) / \beta} \log ^{\mathrm{l} / \beta}(1 /(1-\rho))\right) \cdot \mathrm{M}(\rho \mathrm{r}) \\
& \geq \exp \left(-\mathrm{K}(1-\rho)^{\left.-(1-\beta) / \beta-\varepsilon^{\prime}\right) \cdot \mathrm{M}(\rho \mathrm{r})}\right.
\end{aligned}
$$

are valid when $\rho_{0}<\rho<1$.

Suppose the theorem is false. Then for each $\varepsilon(0<\varepsilon<1)$, there is a sequence of values of $r_{0}$ approaching 1 with 


$$
\left(\log M\left(r_{0}\right)\right)^{\gamma}>\frac{4}{\varepsilon\left(1-r_{0}\right)} \quad\left(\gamma<\frac{\beta}{1-\beta}\right)
$$

By Lemma 1, there exists for each $r_{0}$ an $r^{\prime}$ such that

$$
\log M\left(r^{\prime}+\frac{1}{\left(\log M\left(r^{\prime}\right)\right)^{\gamma}}\right)<(1+\varepsilon)^{1 / \gamma} \log M\left(r^{\prime}\right) \leq(1+\eta) \log M\left(r^{\prime}\right)
$$

Let

$$
r=r^{\prime}+\frac{1}{\left(\log M\left(r^{\prime}\right)\right)^{\gamma}} \quad \text { and } \quad \rho=\frac{\mathbf{r}^{\prime}}{r}
$$

Since

$$
\frac{1}{\left(\log M\left(r^{\prime}\right)\right)^{\gamma}} \leq \frac{1}{\left(\log M\left(r_{0}\right)\right)^{\gamma}}<\frac{\varepsilon\left(1-r_{0}\right)}{4}
$$

we find with the aid of Lemma 1 that

$$
\mathrm{r}_{0}<\mathrm{r}<\mathrm{r}^{\prime}+\frac{\varepsilon\left(1-\mathrm{r}_{0}\right)}{4}<\frac{1+\mathrm{r}_{0}}{2}+\frac{\varepsilon\left(1-\mathrm{r}_{0}\right)}{4}<\mathrm{r}_{0}+\frac{3}{4}\left(1-\mathrm{r}_{0}\right) \text {. }
$$

Consequently

(10) $(\log M(r))^{\gamma}>\left(\log M\left(r_{0}\right)\right)^{\gamma}>\frac{4}{\varepsilon\left(1-r_{0}\right)} \geq \frac{1 / \varepsilon}{1-\left(r_{0}+\frac{3}{4}\left(1-r_{0}\right)\right)}>\frac{1}{\varepsilon(1-r)}$. Also,

$$
(1-\rho)>\mathbf{r}-\mathbf{r}^{\prime}=\frac{1}{\left(\log \mathrm{M}\left(\mathrm{r}^{\prime}\right)\right)^{\gamma}}>\frac{1}{(\log \mathrm{M}(\mathrm{r}))^{\gamma}}
$$

Therefore, by (8) and (9),

$$
\sup _{0 \leq \mathrm{u} \leq \mathrm{r}}\left|\mathrm{f}\left(\mathrm{ue}^{\mathrm{i} \theta}\right)\right| \geq \exp \left(-\mathrm{K}(\log \mathrm{M}(\mathrm{r}))^{\gamma\left((1-\beta) / \beta+\varepsilon^{\prime}\right)}\right) \cdot(\mathrm{M}(\mathrm{r}))^{1 /(1+\eta)} .
$$

Choose $\varepsilon^{\prime}$ so small that $\mathbf{E}=\gamma\left((1-\beta) / \beta+\varepsilon^{\prime}\right)<1$. Then

$$
\begin{aligned}
\sup _{0 \leq u \leq r}\left|f\left(u e^{i \theta}\right)\right| & \geq \exp \left(\frac{\log M(r)}{1+\eta}-K(\log M(r))^{E}\right) \\
& \geq \exp \left(\left(1-\varepsilon_{0}\right) \log M(r)\right)=M(r)^{1-\varepsilon_{0}},
\end{aligned}
$$

where $\varepsilon_{0}<\frac{1}{8}$, say, for $r$ sufficiently near 1 .

For these values of $r$, we shall see (using an argument that originated with Polya) that the region of $|z| \leq r$ in which

$$
|\mathrm{f}(\mathrm{z})| \geq(\mathrm{M}(\mathrm{r}))^{1-2 \varepsilon_{0}}
$$

encircles the origin. If it did not, there would exist a curve c from the origin to $|z|=r$ along which $|f(z)|<M(r)^{1-2 \varepsilon_{0}}$. Let $z_{0}$ be the first point at which $c$ 
intersects $|z|=r$, and consider the radius $R$ joining the origin to $z_{0}$. Let $\bar{c}$ be the reflection of $c$ across $R$. Since $|f(z)| \leq M(r)$ on $\bar{c}$, the inequality

$$
\left|f\left(z^{\prime}\right)\right|<M(r)^{1 / 2} \cdot M(r)^{\left(1-2 \varepsilon_{0}\right) / 2}=M(r)^{1-\varepsilon_{0}}
$$

holds for all $z^{\prime}$ on $R$. This contradicts (11). Hence $|f(z)| \geq M(r)^{1-2 \varepsilon_{0}}$ on some curve $\mathrm{K}$ encircling the origin.

Let

$$
P(z)=\prod_{1}^{n}\left(1-z / z_{k}\right)
$$

where $\left\{z_{k}\right\}$ is the set of zeros of $f(z)$ in $|z|<r$, and set

$$
\mathrm{e}^{\phi(z)}=\frac{\mathbf{f}(\mathrm{z})}{\mathrm{P}(\mathrm{z})}
$$

Clearly, for $\mathrm{z}$ on $\mathrm{K}$,

$$
\log |P(z)|=\sum_{1}^{n} \log \left|1-\frac{z}{z_{k}}\right| \leq \sum_{1}^{n} \log \left(1+\left|\frac{z}{z_{k}}\right|\right) \leq K_{0} \frac{1}{(1-r)^{\lambda}}
$$

where $K_{0}$ is a positive constant independent of $r$. We note that

$$
\mathrm{K}_{0} \frac{1}{(1-\mathrm{r})^{\lambda}}=\frac{\mathrm{K}}{\varepsilon^{1 / \gamma}} \frac{(1-\mathrm{r})^{1 / \gamma-\lambda}}{(1-\mathrm{r})^{1 / \gamma}}
$$

where $K_{1}$ is a positive constant independent of $r$. Taking values of $r_{0}$ (and thus of the associated $r$ ) nearer 1 , if necessary, we see that (10) and (13) together with the fact that $1 / \gamma>\lambda$ now yield

$$
\log |\mathrm{P}(\mathrm{z})| \leq \frac{1}{2} \cdot \frac{1}{(\varepsilon(1-\mathrm{r}))^{1 / \gamma}}<\frac{1}{2} \cdot \log \mathrm{M}(\mathrm{r})
$$

for $z$ on $K$. Hence, with the aid of (12), the minimum modulus theorem, and the definition of $\mathrm{K}$, we have a sequence of values of $r$ approaching 1 for which we know the inequality

$$
\mathrm{e}^{\phi(0)}>\mathrm{M}(\mathrm{r})^{1 / 4}
$$

But this is clearly impossible.

\section{PROOFS OF THE LEMMAS}

Proof of Lemma 1. Suppose (5) is false for $\mathrm{x}_{0}$. Define $\mathrm{x}_{1}$ to be the lower bound of all $\mathrm{x}>\mathrm{x}_{0}+1 / \phi\left(\mathrm{x}_{0}\right)$ for which the inequality is false. Inductively, define $\mathrm{x}_{\mathrm{n}}$ to be the lower bound of all $x>x_{n-1}+1 / \phi\left(x_{n-1}\right)$ for which the inequality is false. Continuity ensures the falseness of the inequality for the $x_{i}$ also. Then the total measure of the set of $x$ in $\left[x_{0}, 1\right)$ for which (5) is false is majorized by 


$$
\sum_{n=0}^{\infty} \frac{1}{\phi\left(\mathrm{x}_{\mathrm{n}}\right)}<\frac{1}{\phi\left(\mathrm{x}_{0}\right)} \sum_{\mathrm{n}=0}^{\infty}\left(\frac{1}{1+\varepsilon}\right)^{\mathrm{n}}=\frac{1}{\phi\left(\mathrm{x}_{0}\right)}\left(\frac{1+\varepsilon}{\varepsilon}\right) \text {. }
$$

From (4) we obtain the inequality

$$
\frac{1}{\phi\left(\mathrm{x}_{0}\right)}\left(\frac{1+\varepsilon}{\varepsilon}\right)<\frac{1-\mathrm{x}_{0}}{2}
$$

this completes the proof.

Proof of Lemma 2. Consider the function

$$
G(z)=\frac{\varepsilon_{0}}{(z+1)^{2}} \prod_{k=0}^{\infty} \frac{m_{k}+1-z}{m_{k}+1+z},
$$

where the $m_{i}$ are the midpoints of the segments $\left(n_{k}, n_{k+1}\right)$ for which $\varepsilon_{k}$ and $\varepsilon_{k+1}$ are distinct. By (2), we see that $\sum_{1 / n_{k}<\infty}$, which implies $\sum 1 / \mathrm{m}_{\mathrm{k}}<\infty$. Hence $\mathrm{G}(\mathrm{z})$ defines an analytic function in $\Re \mathrm{z}>-1$.

A Laplace inversion theorem (see Churchill [2, p. 178]) implies that $G(z)$ is the Laplace transform of the function

$$
g\left(e^{-s}\right)=\frac{1}{2 \pi \dot{i}} \int_{c_{0}-i \infty}^{c_{0}+i \infty} e^{z s} G(z) d z,
$$

where $c_{0}$ is any real number greater than -1 . Therefore the function

$$
g(t)=\frac{1}{2 \pi} \int_{-\infty}^{+\infty} e^{-i y \log t} G(i y) d y
$$

satisfies (i), with $A_{k}=\left|G\left(n_{k}+1\right)\right|$.

(ii) follows easily, because

$$
\int_{0}^{1}|g(t)| d t \leq \frac{1}{2 \pi} \int_{-\infty}^{+\infty}|G(i y)| d y=\frac{1}{2 \pi} \int_{-\infty}^{+\infty}\left(\frac{1}{1+y^{2}}\right) \prod_{k=0}^{\infty}\left|\frac{m_{k}+1-i y}{m_{k}+1+i y}\right| d y \leq \frac{1}{2} .
$$

To obtain (iii), we proceed to estimate $\left|G\left(n_{q}+1\right)\right|$ for fixed $q>0$. We note that for positive $z$

$$
|G(z)| \geq \frac{1}{|z+1|^{2}}\left\{\prod_{k=0}^{\infty}\left|\frac{\frac{1}{2}\left(n_{k}+n_{k+1}\right)+1-z}{\frac{1}{2}\left(n_{k}+n_{k+1}\right)+1+z}\right|\right\}
$$

so that

$$
\left|G\left(n_{q}+1\right)\right| \geq \frac{1}{\left(n_{q}+2\right)^{2}} \prod_{k=0}^{\infty}\left|\frac{n_{k}+n_{k+1}-2 n_{q}}{n_{k}+n_{k+1}+4+2 n_{q}}\right|
$$


Setting $\mu_{\mathrm{k}}=\mathrm{n}_{\mathrm{k}}+\mathrm{n}_{\mathrm{k}+\mathrm{l}}$, we estimate separately the terms of the products $\Pi_{1}, \Pi_{2}$, $\Pi_{3}$ with $\mu_{\mathrm{k}}>4 \mathrm{n}_{\mathrm{q}}, 2 \mathrm{n}_{\mathrm{q}}<\mu_{\mathrm{k}} \leq 4 \mathrm{n}_{\mathrm{q}}$, and $\mu_{\mathrm{k}}<2 \mathrm{n}_{\mathrm{q}}$, respectively.

We have the inequalities

$$
\begin{aligned}
\Pi_{1} & =\prod_{\mu_{\mathrm{k}}>4 \mathrm{n}_{\mathrm{q}}}\left(\frac{1-\frac{2 \mathrm{n}_{\mathrm{q}}}{\mu_{\mathrm{k}}}}{1+\frac{2\left(\mathrm{n}_{\mathrm{q}}+2\right)}{\mu_{\mathrm{k}}}}\right) \geq \prod_{\mu_{\mathrm{k}}>4 \mathrm{n}_{\mathrm{q}}} \exp \left(-\frac{4 \mathrm{n}_{\mathrm{q}}}{\mu_{\mathrm{k}}}-\frac{2\left(\mathrm{n}_{\mathrm{q}}+2\right)}{\mu_{\mathrm{k}}}\right) \\
& \geq \exp \left(-\left(6 \mathrm{n}_{\mathrm{q}}+4\right) \sum_{\mathrm{s}^{\prime}} \frac{1}{\mu_{\mathrm{k}}}\right),
\end{aligned}
$$

where $S^{\prime}=\left\{k \mid \mu_{k}>4 n_{q}\right\}$. But since $\mu_{k}=n_{k}+n_{k+1}<2 n_{k+1}$, $S^{\prime}$ is a subset of $\mathrm{S}=\left\{\mathrm{k} \mid \mathrm{n}_{\mathrm{k}+1}>2 \mathrm{n}_{\mathrm{q}}\right\}$, so that

$$
2 \sum_{s^{\prime}} \frac{1}{\mu_{\mathrm{k}}} \leq \sum_{\mathrm{s}} \frac{1}{\mathrm{n}_{\mathrm{k}}} \leq \int_{\mathrm{n}_{\mathrm{q}}}^{\infty} \frac{\mathrm{dN}^{0}(\mathrm{u})}{\mathrm{u}}=-\frac{\mathrm{N}^{0}\left(\mathrm{n}_{\mathrm{q}}\right)}{\mathrm{n}_{\mathrm{q}}}+\int_{\mathrm{n}_{\mathrm{q}}}^{\infty} \frac{\mathrm{N}^{0}(\mathrm{u})}{\mathrm{u}^{2}} \mathrm{du} .
$$

Therefore, since (2) implies $N^{0}(u)<K_{1} u^{1-\beta}$, we find by combining (14) and (15) that

$$
\Pi_{1} \geq \exp \left(-\mathrm{K}_{2} \mathrm{n}_{\mathrm{q}}^{1-\beta}\right)
$$

(the $\mathrm{K}_{\mathrm{i}}$ denote constants).

Next we observe that

$$
\Pi_{2} \geq\left(6 n_{q}+4\right)^{-\left(K_{1}\left(2 n_{q}\right)^{1-\beta}-1-(q-1)\right)}
$$

because $\mathrm{n}_{\mathrm{k}+1} \leq 2 \mathrm{n}_{\mathrm{q}}$ implies $\mathrm{k}+1<\mathrm{K}_{1}\left(2 \mathrm{n}_{\mathrm{q}}\right)^{1-\beta}$, by (2).

Finally,

$$
\begin{aligned}
\Pi_{3} & =\prod_{k=0}^{q-1}\left(\frac{2 n_{q}-\left(n_{k}+n_{k+1}\right)}{2 n_{q}+4+\left(n_{k}+n_{k+1}\right)}\right) \geq\left\{\prod_{k=0}^{q-2}\left(\frac{2 n_{q}-2 n_{k+1}}{2 n_{q}+4+2 n_{k+1}}\right)\right\} \cdot \frac{n_{q}-n_{q-1}}{3 n_{q}+n_{q-1}+4} \\
& \geq\left\{\prod_{k=0}^{q-2}\left(\frac{n_{q}-n_{k+1}}{n_{q}+2+n_{k+1}}\right)\right\} \cdot \frac{n_{q}-n_{q-1}}{3 n_{q}+n_{q-1}+4} \geq\left(2 n_{q}+2\right)^{-(q-1)} \cdot\left(4 n_{q}+4\right)^{-1}
\end{aligned}
$$

From (16), (17), and (18) it follows that

$$
\left|\mathrm{G}\left(\mathrm{n}_{\mathrm{q}}+1\right)\right| \geq\left(10 \mathrm{n}_{\mathrm{q}}\right)^{-\left(\mathrm{K}_{1} 2^{1-\beta}+2\right) \mathrm{n}_{\mathrm{q}}^{1-\beta}} \cdot \exp \left(-\mathrm{K}_{2} \mathrm{n}_{\mathrm{q}}^{1-\beta}\right) \geq\left(\mathrm{K}_{3} \mathrm{n}_{\mathrm{q}}\right)^{-\mathrm{K}_{4} \mathrm{n}_{\mathrm{q}}^{1-\beta}} .
$$

Let

then

$$
\mathrm{h}(\mathrm{u})=\left(\mathrm{K}_{3} \mathrm{u}\right)^{-\mathrm{K}_{4} \mathrm{u}^{1-\beta}} \cdot \frac{1}{\rho^{\mathrm{u}}} \quad(\mathrm{u}>0)
$$




$$
\frac{h^{\prime}(u)}{h(u)}=-(1-\beta) u^{-\beta} K_{4} \log \left(K_{3} u\right)-K_{4} u^{-\beta}+\log \frac{1}{\rho}
$$

Therefore, for $u>u_{0}$, we find that inf $h(u)$ occurs when

$$
\log \frac{1}{\rho} \approx(1-\beta) \mathrm{K}_{4} \mathrm{u}^{-\beta}(\log \mathrm{u})
$$

That is, setting $\log (1 / \rho)=s$, we have the estimate

$$
\mathrm{u} \approx \mathrm{K}_{5} \mathrm{~s}^{-1 / \beta}\left(\log \frac{1}{\mathrm{~s}}\right)^{1 / \beta}
$$

Returning then to $h(u)$, we see that

$$
\begin{aligned}
\inf _{\mathrm{k}} \mathrm{A}_{\mathrm{k}} \rho^{-\mathrm{n}_{\mathrm{k}}} & >\exp \left(-\mathrm{Ks}^{-(1-\beta) / \beta} \log ^{1 / \beta}(1 / \mathrm{s})\right) \\
& >\exp \left(-\mathrm{K}(1-\rho)^{-(1-\beta) / \beta} \log ^{1 / \beta}(1 /(1-\rho))\right) .
\end{aligned}
$$

Remarks. The method of proof above yields similar results for other assumptions on gaps and corresponding growth. In addition, the proof shows the nonexistence of finite asymptotic paths for such functions.

The theorem of Sunyer I. Balaguer previously mentioned can be stated by replacing (3) in Theorem 1 by the condition

$$
\alpha>\max \left(\lambda, \frac{(1-\beta)^{1 / 2}+(1-\beta)}{\beta}\right) .
$$

The method of proof of Sunyer I. Balaguer's assertion is considerably more complicated than that of Theorem 1 .

\section{REFERENCES}

1. M. Biernacki, Sur les zéros des polynomes et sur les fonctions entières dont le développement Taylorien présente des lacunes, Bull. Sci. Math. (2) 69 (1945), 197-203.

2. R. V. Churchill, Operational mathematics, Second Edition, McGraw-Hill, New York, 1958.

3. W. H. J. Fuchs, Proof of a conjecture of G. Pólya concerning gap series, Illinois J. Math. 7 (1963), 661-667.

4. W. K. Hayman, Meromorphic functions, Clarendon Press, Oxford, 1964.

5. T. Kövari, A gap-theorem for entire functions of infinite order, Michigan Math.

J. 12 (1965), 133-140.

6. C. Pommerenke, Lacunary power series and univalent functions, Michigan Math.

J. 11 (1964), 219-223. 
7. F. Sunyer I. Balaguer, Sur la substitution d'une valeur exceptionnelle par une propriété lacunaire, Acta Math. 87 (1952), 17-31.

8. M. Weiss and G. Weiss, On the Picard property of lacunary power series, Studia Math. 22 (1962-63), 221-245.

Cornell University

and

Northern Illinois University 
